\title{
Metastatic mixed gestational trophoblastic tumour of the uterus: A case report
}

\author{
Fontanella ${ }^{1 *}$, Manganiello CAT ${ }^{1}$, Borrelli $S^{2}$, Natalizi $S^{3}$, Brogna $B^{4}$, Catarinella $V^{5}$ and De Blasio $A^{5}$ \\ ${ }^{1}$ Radiology Department, Ospedale Sacro Cuore di Gesù FBF, Benevento, Italy \\ ${ }^{2}$ Hillman Cancer Center UPMC Villa Maria, Mirabella Eclano, Italy \\ ${ }^{3}$ Pathology Division, Ospedale San Pietro FBF, Rome, Italy \\ ${ }^{4}$ Radiology Department, A.O.R.N. S.G. Moscati, Avellino, Italy \\ ${ }^{5}$ Obstetrics Division, Ospedale Sacro Cuore di Gesù FBF, Benevento, Italy
}

\section{Introduction}

A mixed gestational trophoblastic neoplasia (GTN) is a very rare, histologically heterogeneous form of tumour, generally made up by choriocarcinoma and/or placental site trophoblastic tumour (PSTT) and/or epithelioid trophoblastic tumour (ETT) [1-3].

The most common combination is a choriocarcinoma mixed with an ETT and/or PSTT [4].

Considering the diagnosis of pure forms of GTNs is challenging itself - PSST, for example, has a prevalence of $<1 / 1000000$, with less than 200 cases described in literature - mixed forms can be extremely hard to detect [5].

GTN present in women during the reproductive age and very rarely after menopause, generally with irregular metrorrhagia, weeks, months or years after a regular pregnancy, spontaneous miscarriage or voluntary termination; sometimes, GTNs might present with unexplained metastases, which often affect the lungs, in the weeks and months after normal childbirth or ectopic pregnancy [1].

Even though vaginal bleeding is of course the most common, often only symptom, serum $\beta$-HCG levels are typically elevated, not exceeding $2500 \mathrm{mIU} / \mathrm{ml}$ in most cases.

Mixed gestational trophoblastic neoplasms are treated with hysterectomy and careful lymph node dissection; metastatic cases are treated with adjuvant chemotherapy [6].

\section{Case description}

A 34 year-old Caucasian woman was referred to our Obs/Gyn division, presenting with unexplained metrorrhagia, almost 4 months after normal childbirth. All laboratory tests were unremarkable, except for elevated serum $\beta$-HCG (4333 IU/L) and CA-125 levels (366 KU/L). The patient was then referred to our Radiology unit, for a Chest X-ray and abdominal ultrasound evaluation; a dishomogeneously hypoecoic uterine mass $(4.4 \times 4.2 \mathrm{~cm}$, Figure 1) was noted. Except for this finding, the initial imaging tests were otherwise unremarakable. The patient was immediately scheduled for a contrast-enhanced Pelvic MRI evaluation, that confirmed the presence of a dishomogenous, irregularly round mass $(4.5 \times 4.3 \times 3.6 \mathrm{~cm}$, Figures $2-5)$, in the endometrial cavity but protruding outwards, eroding the myometrium, the latter barely recognisable at the right lateral aspect of the uterine fundus. The postcontrast acquisitions showed the mass to be highly vascular, almost reaching the outlying sierosa.

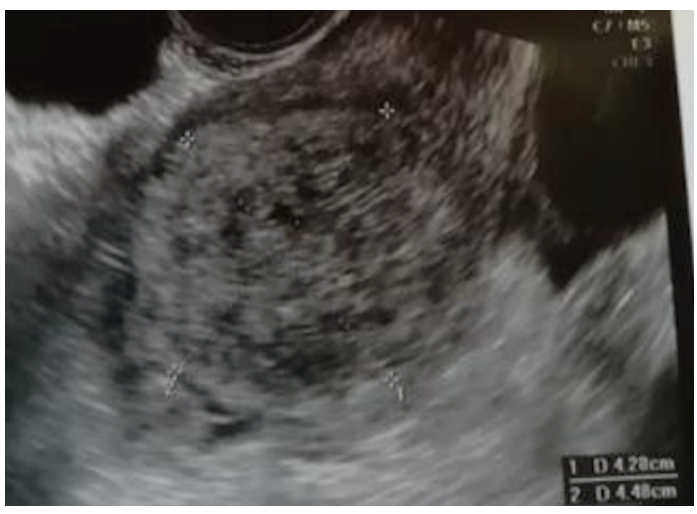

Figure 1. Ultrasound appearance of the mixed PSTT/Choriocarcinoma GTN. A rounded, heterogeneously ecoic mass is noted within the endometrial cavity

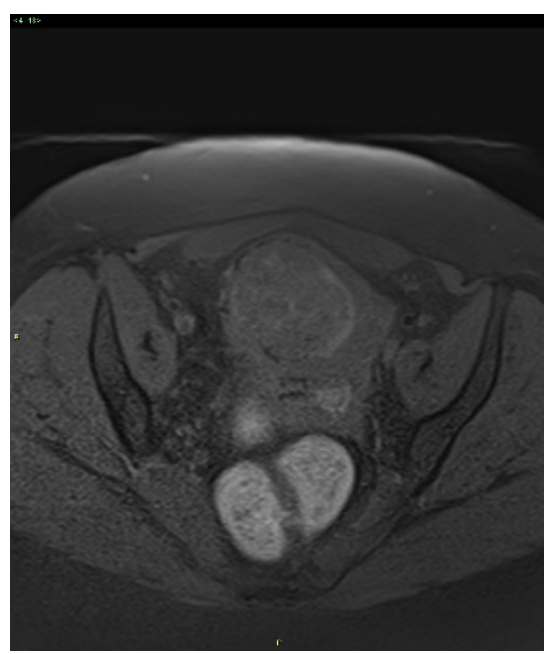

Figure 2. MRI Axial pre-contrast fat-sat $\mathrm{T} 1$ weighted sequence shows a rounded, dishomogeneously intense mass within the endometrial cavity; hyperintense spots within and around the mass are suggestive for haemorrhage. Infiltration of the right cranial aspect of the fundic myometrium is noted

${ }^{\star}$ Correspondence to: Fontanella G, Radiology Department, Ospedale Sacro Cuore di Gesù FBF, Benevento, Italy, E-mail: giovanni.fontanella@hotmail.com

Received: February 24, 2021; Accepted: March 04, 2021; Published: March 08, 2021 


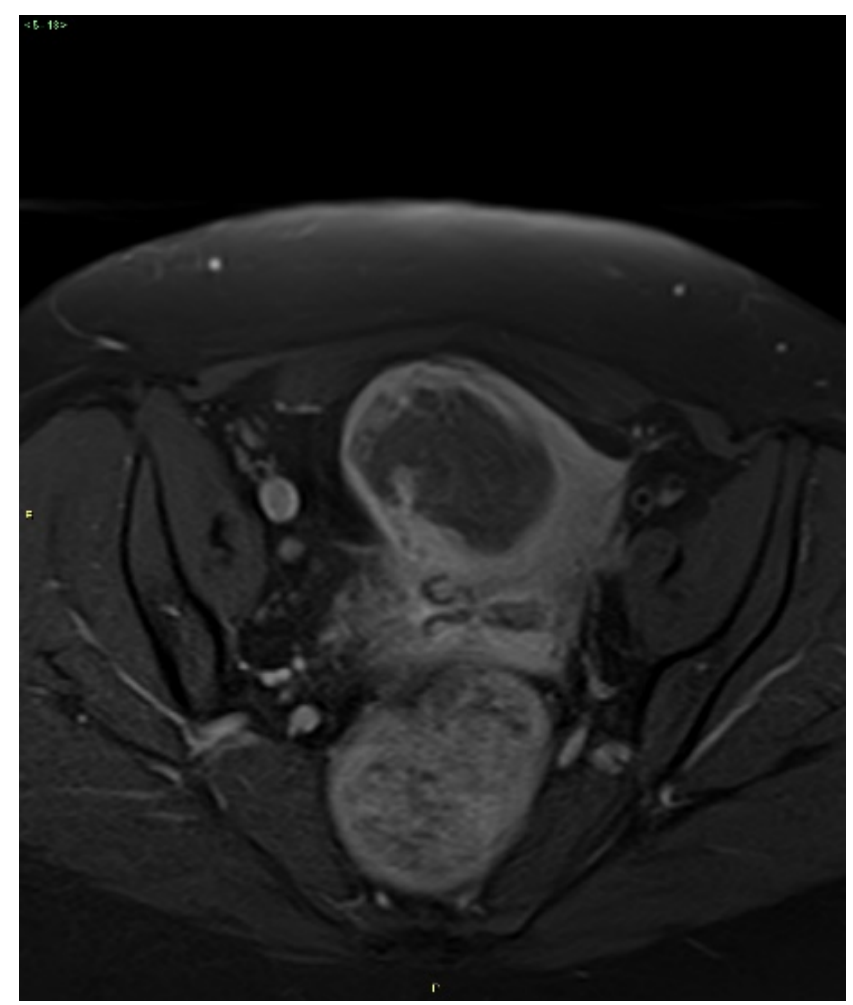

Figure 3. MRI Axial post-contrast fast-sat T1 weighted sequence shows dishomogeneous enhancement of the mass, which is prevalently peripheral. Enhancement spots within the mass are found, too

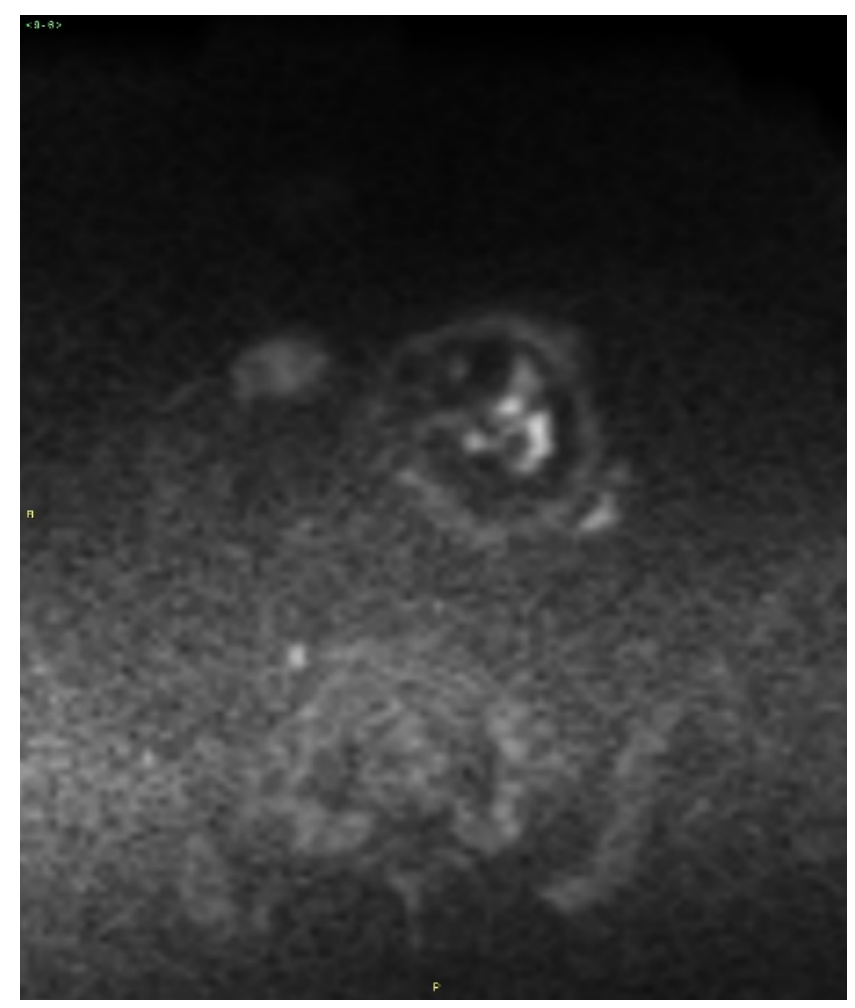

Figure 4. MRI Diffusion weighted images (DWI) show hyperintense spots within the mass, highly suggestive for pathologic hypercellularity. The vast majority of the mass is hypointense, due to the high presence of necrosis. No pathologic lymph nodes are found

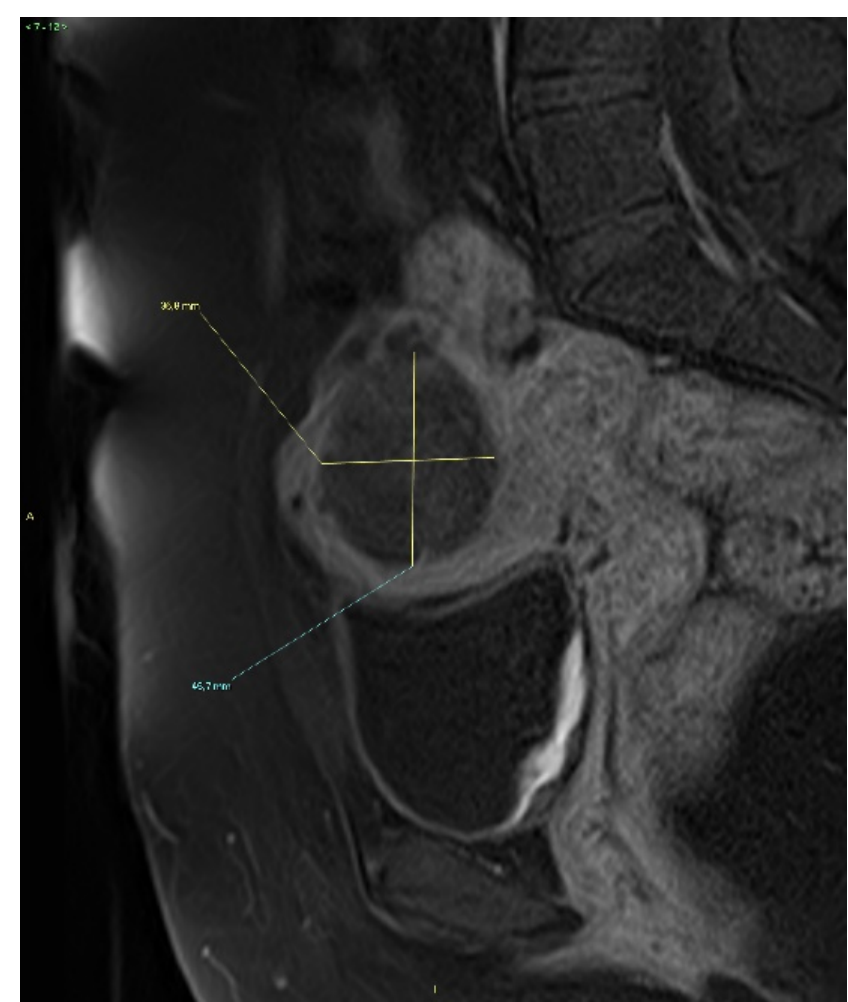

Figure 5. MRI Sagittal post-contrast fat-sat $\mathrm{T} 1$ weighted sequence clearly shows peripheral contrast enhancement and the infiltration of the right cranial aspect of the fundic myometrium

DWI/ADC sequences showed the presence of areas of pathologically restricted water diffusivity, highly suspect for hypercellularity.

No significative locoregional lymph node localizations were noted.

After multidisciplinary evaluation, considering the clinical history and recent pregnancy, the unexplained, high serum $\beta$-HCG levels and the MRI findings, a diagnosis of gestational trophoblastic neoplasia, namely PSST, was suspected.

The patient was then scheduled for a Total Body CECT, which was carried out three days later, to rule out the presence of distant disease. The pelvic findings were, of course, totally confirmed; a slight increase in vascularization was noted, albeit the MRI exam was executed only three days before. Even though abdomen, pelvis and head, except for the mass, were completely unremarkable, the presence of small $(<0.6 \mathrm{~cm})$, numerous $(>60)$, rounded lung nodules, compatible with metastases, was noted.

A re-evaluation of the initial Chest X-ray, in light of the CECT findings, confirmed an unremarkable examination, the lung nodules probably too small to be radiographically evident.

Hysterectomy, with prudential lymph node dissection, was subsequently performed; after that, the patient was referred to our Oncology Department and is currently undergoing adjuvant chemotherapy.

The pathology report highlighted the presence of necrosis and a biphasic pattern in the mass, with inibine+/panCK+/GATA3+/PLAP+/ $\beta-\mathrm{HCG}+/ \mathrm{p} 63$ - nodules, showing traits compatible with elements of the intermediate trophoblast and $\beta$-HCG+/PLAP- nodules, showing traits compatible with elements of syncytiotrophoblast. 
Ki-67 replication index was 30\% for the first set of nodules, and $90 \%$ for the latter. The two histological patterns were respectively compatibile with PSTT and choriocarcinoma, which combine to form a mixed gestational trophoblastic neoplasia (Figures 6-9).

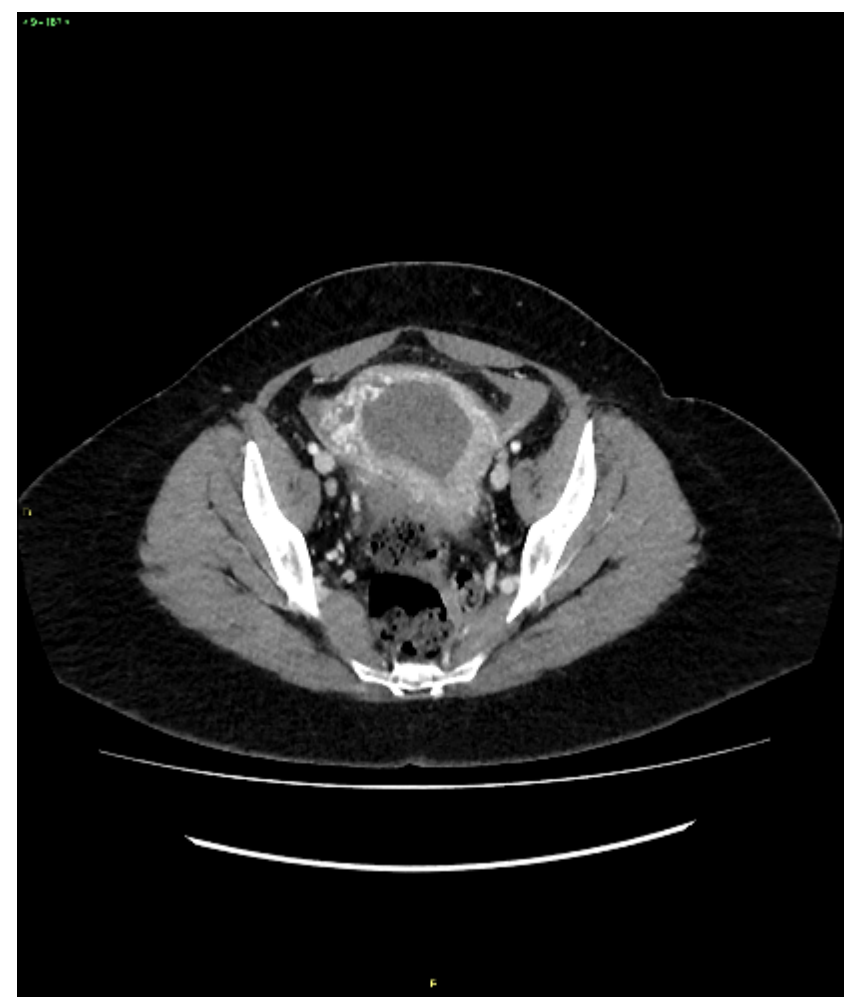

Figure 6. Post-contrast arterial phase CT appearance of the mixed PSTT/Choriocarcinoma GTN. Intense peripheral contrast enhancement of the mass, with infiltration of the myometrium towards the sierosa

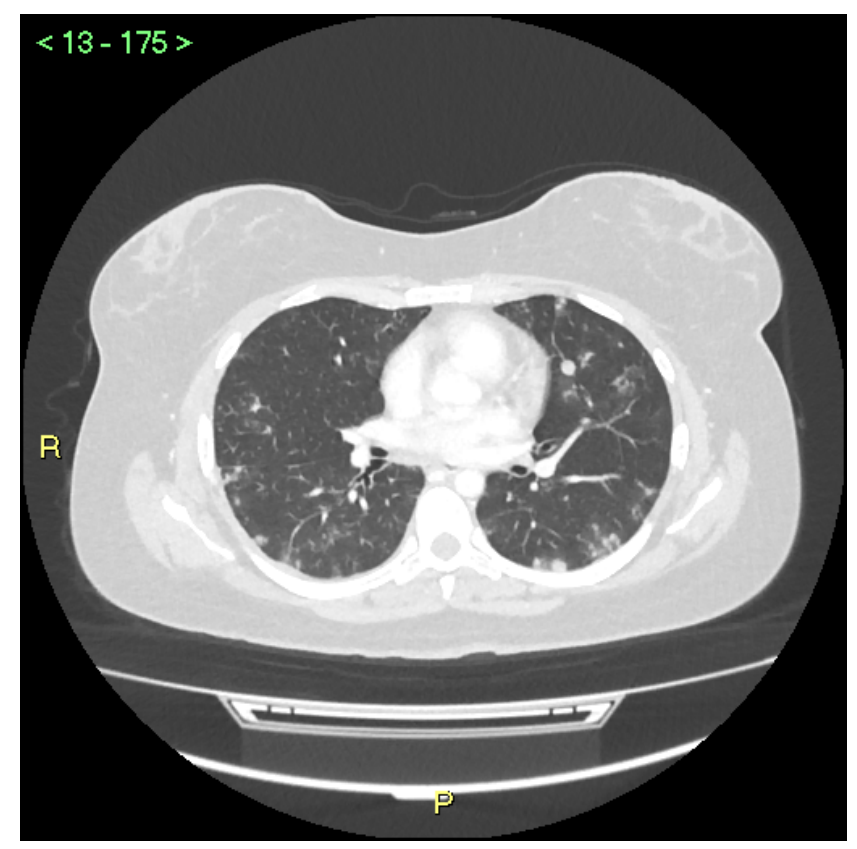

Figure 7. CECT - multiple $(>60)$ millimetric rounded metastatic nodules in both lungs. No pleural or pericardial effusions

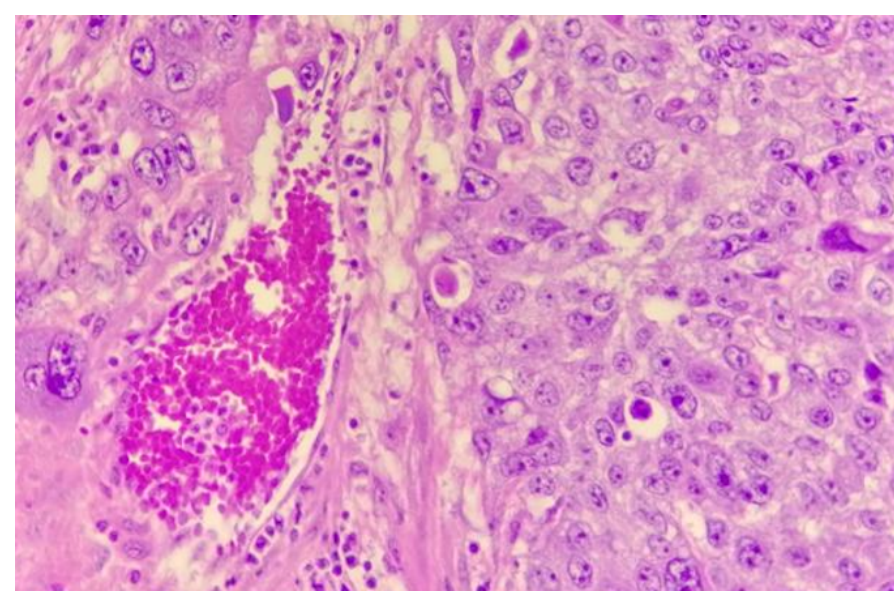

Figure 8. Pathologic features of the mixed PSTT/Choriocarcinoma GTN. The presence of plurinucleated elements with clear cytoplasm is suggestive for syncytiotrophoblast and choriocarcinoma. Haemorrhage, necrosis are noted too

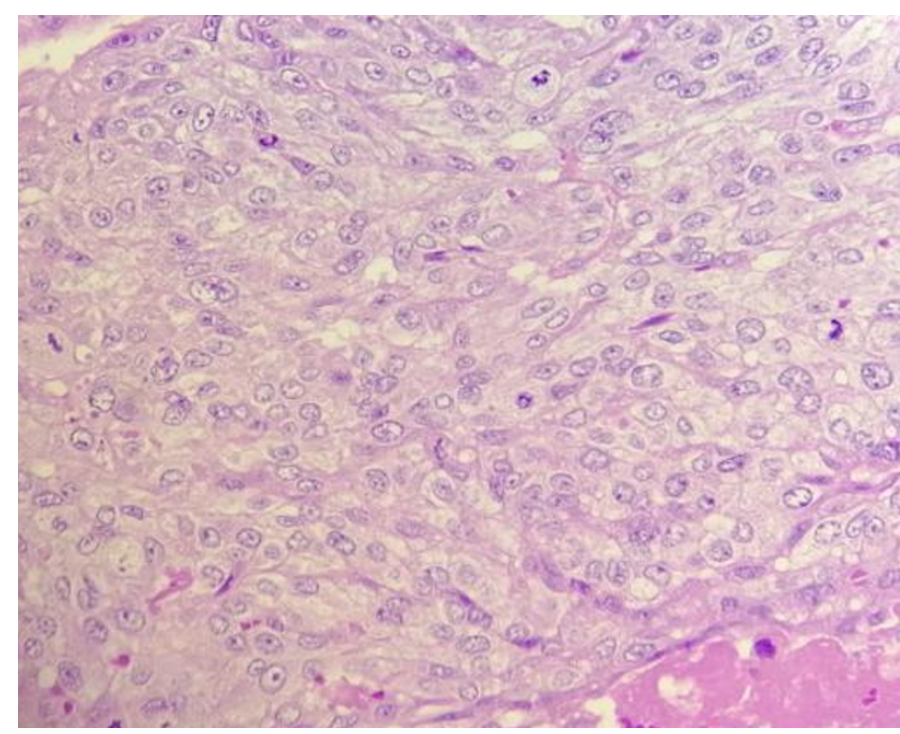

Figure 9. Pathologic features of the mixed PSTT/Choriocarcinoma GTN. Clusters of moderately pleiomorphic, mononucleated elements suggestive for intermediate trophoblast and PSTT, with a high mitotic index ( $>5$ mitoses/field)

\section{Discussion}

The diagnostic process, from symptom to surgery, was unusually straightforward in our case. The presence of unexplained metrorrhagia and elevated serum $\beta$-HCG levels, even though not as elevated as one would expect in a pure choriocarcinoma $(>10000-20000 \mathrm{mIU} / \mathrm{ml})$ [3], were extremely suspect, especially after pregnancy in a relatively young patient. The presence of the mass led us, then, to consider other forms of gestational trophoblastic tumours in the differential, especially PSTT. The difficulty in diagnosing a mixed GTN resides in the fact that the slightly elevated $\beta$-HCG is an ambiguous lab finding, albeit not compatible with a pure choriocarcinoma and the fact that there is no fixed MRI pattern for GTNs, both pure and mixed; they tend to be infiltrative heterogeneously necrotic masses, extending to the myometrium and beyond, with some cystic colliquation and variable vascularization, which tends to be extreme in the case of choriocarcinoma, leading to hematogenous metastases. In our case, the MRI findings were compatible with a GTN/choriocarcinoma, lab 
findings were more suggestive for PSTT or ETT; pathology confirmed the presence of both choriocarcinoma and PSTT, justifying the 'mixed' clinical and imaging picture. The presence of the choriocarcinoma component is most likely responsible for the quick hematogenous spread to the lungs of the disease and, unfortunately, for the worse prognosis for our patient, considering that many cases with pure PSTT tend to be local and treated with hysterectomy only; however, a few metastatic cases have shown to be responding to high-dose chemotherapy, in addiction to hysterectomy [6].

\section{Conclusion}

Even though pure and mixed GTNs are a quite rare disease, the presence of vaginal bleeding/metrorrhagia, plus elevated serum $\beta$-HCG, should prompt the suspect in the differential. Pelvic, contrast-enhanced MRI is the method of choice for regional disease staging; total-body CECT should always be added, too, to exclude the spread of the disease to distant sites, especially to the lungs. Treatment can be by hysterectomy only or by hysterectomy plus chemotherapy, when in presence of metastases or in selected, high-risk patients with local disease.

\section{References}

1. Shih IeM (2007) Gestational trophoblastic neoplasia-pathogenesis and potential therapeutic targets. Lancet Oncol 8: 642-650. [Crossref]

2. Shih IM, Kurman RJ (1998) Epithelioid trophoblastic tumor: a neoplasm distinct from choriocarcinoma and placental site trophoblastic tumor simulating carcinoma. Am J Surg Pathol 22: 1393-1403. [Crossref]

3. Shen DH, Khoo US, Ngan HY, Ng TY, Chau MT, et al. (2003) Coexisting epithelioid trophoblastic tumor and choriocarcinoma of the uterus following a chemoresistant hydatidiform mole. Arch Pathol Lab Med 127: e291-293. [Crossref]

4. Sung WJ, Shin HC, Kim MK, Kim MJ (2013) Epithelioid trophoblastic tumor: clinicopathologic and immunohistochemical analysis of three cases. Korean J Pathol 47: 67-73. [Crossref]

5. Luk WY, Friedlander MA (2013) Fibroid or cancer? a rare case of mixed choriocarcinoma and epithelioid trophoblastic tumour. Case Rep Obstet Gynecol 2013: 492754. [Crossref]

6. Knox S, Brooks SE, Wong-You-Cheong J, Ioffe O, Meisenberg B, et al. (2002) Choriocarcinoma and epithelial trophoblastic tumor: successful treatment of relapse with hysterectomy and high-dose chemotherapy with peripheral stem cell support: a case report. Gynecol Oncol 85: 204-208. [Crossref]

Copyright: (C2021 Fontanella G. This is an open-access article distributed under the terms of the Creative Commons Attribution License, which permits unrestricted use, distribution, and reproduction in any medium, provided the original author and source are credited. 